\title{
Microsatellite loci in Japanese quail and cross-species amplification in chicken and guinea fowl
}

\author{
Boniface Baboreka KAYANG ${ }^{a}$, Miho InOUE-MUrayAmA ${ }^{\mathrm{b} *}$, \\ Takuya Hoshi $^{\mathrm{b}}$, Koji MATSUO ${ }^{\mathrm{b}}$, Hideaki TAKAHASHI ${ }^{\mathrm{c}}$, \\ Mitsuru Minezawa ${ }^{\mathrm{c}}$, Makoto Mizutani ${ }^{\mathrm{d}}$, Shin'ichi Ito ${ }^{\mathrm{b}}$ \\ ${ }^{a}$ The United Graduate School of Agricultural Science, \\ Gifu University, Gifu 501-1193, Japan \\ ${ }^{\mathrm{b}}$ Faculty of Agriculture, Gifu University, Gifu 501-1193, Japan \\ ${ }^{c}$ National Institute of Agrobiological Sciences, Tsukuba 305-8602, Japan \\ ${ }^{\mathrm{d}}$ Laboratory Animal Research Station, Nippon Institute for Biological Science, \\ Kobuchizawa 408-0041, Japan
}

(Received 28 June 2001; accepted 10 September 2001)

\begin{abstract}
In line with the Gifu University's initiative to map the Japanese quail genome, a total of 100 Japanese quail microsatellite markers isolated in our laboratory were evaluated in a population of 20 unrelated quails randomly sampled from a colony of wild quail origin. Ninety-eight markers were polymorphic with an average of 3.7 alleles per locus and a mean heterozygosity of 0.423 . To determine the utility of these markers for comparative genome mapping in Phasianidae, cross-species amplification of all the markers was tested with chicken and guinea fowl DNA. Amplification products similar in size to the orthologous loci in quail were observed in 42 loci in chicken and 20 loci in guinea fowl. Of the cross-reactive markers, $57.1 \%$ in chicken and $55.0 \%$ in guinea fowl were polymorphic when tested in 20 birds from their respective populations. Five of 15 markers that could cross-amplify Japanese quail, chicken, and guinea fowl DNA were polymorphic in all three species. Amplification of orthologous loci was confirmed by sequencing 10 loci each from chicken and guinea fowl and comparing with them the corresponding quail sequence. The microsatellite markers reported would serve as a useful resource base for genetic mapping in quail and comparative mapping in Phasianidae.
\end{abstract}

Japanese quail / microsatellite loci / chicken / guinea fowl / comparative genetic map

\section{INTRODUCTION}

Microsatellite loci have gained widespread use in genome mapping, phylogenetics, and conservation genetics due to their abundance in eukaryotic

\footnotetext{
* Correspondence and reprints

E-mail: miho-i@cc.gifu-u.ac.jp
} 
genomes, high polymorphism, codominant nature, high reproducibility, and relative ease of scoring by the polymerase chain reaction (PCR). In recent years, genetic linkage maps based on microsatellite markers have been constructed for a number of livestock species including cattle (Bos taurus) [17], sheep (Ovis aries) [9], goats (Capra hircus) [42], and pigs (Sus scrofa) [35]. In the poultry species however, mapping efforts have been slowed by the fewer number of microsatellites present in the avian genome compared to that of mammals [31], and by the large number of cytogenetically similar microchromosomes. In spite of the problems inherent in mapping avian genomes, significant progress has been made for chickens (Gallus gallus) and recently a consensus linkage map of the chicken genome based on Compton [2], East Lansing [4], and Wageningen [11] linkage maps has been published [12]. At present, genetic maps do not exist for other economically important poultry species, including the Japanese quail (Coturnix japonica).

The Japanese quail is valued for its egg and meat, which are enjoyed for their unique flavor [23]. Advantages of small body size, rapid generation turnover, and high egg production [43] make it particularly suited for laboratory research [26], and it has been recommended as a pilot animal for poultry [45]. In the light of this, genetic mapping of this species would be especially desirable if the Japanese quail is to be promoted as a model for poultry. Until now, only two autosomal linkage groups based on plumage color and blood protein markers $[15,16,36]$ and one sex-linked plumage color linkage group [24] have been reported, while DNA markers have not been developed for the Japanese quail. Thus, the quail genome mapping effort was initiated in our laboratory based on the isolation and characterization of microsatellite markers $[14,19]$. As the number of quail microsatellite markers increases, comparative genome analysis of the quail with other closely related species, especially with the more extensively studied chicken, could facilitate the construction of a comparative genetic map in the Phasianidae family, which is our ultimate objective. A step towards achieving this goal would be to uncover cross-reactive markers that could serve as anchor points for future comparative mapping purposes.

Cross-species amplification of microsatellite loci has been reported within closely related livestock species $[3,28,37]$ and has been exploited in the construction of genetic maps for cattle [17], sheep [9], and goats [42] in the Bovidae family. Exchanges of microsatellite markers have also been observed between related avian species $[8,29,30,34]$. In the Phasianidae family, attempts have been made to use the large number of chicken-specific microsatellites available to develop DNA markers for turkeys (Meleagris gallopavo) $[21,22,32,33]$ and Japanese quail [14,27]. However, for comparative mapping purposes, it is also necessary to determine the utility of markers isolated from other Phasianidae species in the chicken. In a preliminary effort, we isolated 50 original quail microsatellite markers and found $46.0 \%$ of them to be polymorphic in two 
unrelated quails [19]. Furthermore, we observed positive amplification for $28.0 \%$ of the loci in the chicken. In this article, we report 50 new quail microsatellite markers and provide a more extensive characterization of all the 100 loci including an evaluation of their usefulness as cross-reactive markers for comparative mapping in chicken and guinea fowl (Numida meleagris), all of which belong to the Phasianidae family.

\section{MATERIALS AND METHODS}

A quail colony maintained at Gifu University was used in this study [14,19]. A population of White Leghorns was sampled from a stock at the Gifu University Experimental Farm, while samples from guinea fowls were obtained from JAFRA TRADING CO., LTD., Ibaragi Prefecture, Japan. Blood was drawn from the jugular vein of quails and by wing venipuncture from White Leghorns and guinea fowls, and DNA was extracted using the QIAamp Blood Kit (Qiagen Inc., CA).

A quail genomic library enriched for the dinucleotide repeat array $(\mathrm{CA} / \mathrm{GT})_{n}$ was constructed [40] and screened following standard procedures, and primers were designed and optimized for PCR as outlined previously [19], with the exception that $1.5 \mathrm{mM} \mathrm{MgCl}{ }_{2}$ concentration was used as the standard to test all markers.

Using the annealing temperature optimized for quail, primer-pairs were tested on chicken and guinea fowl DNA to determine cross-reactive markers. One male and one female of each species were used. Initially, the amplification conditions determined for quail were used for chicken and guinea fowl. Those markers that failed to amplify were further tested at $2.0 \mathrm{mM}$ and $2.5 \mathrm{mM}$ concentrations of $\mathrm{MgCl}_{2}$.

Allelic polymorphism was determined for each marker by performing a PCR on DNA from 20 unrelated quails ( 10 males and 10 females) randomly sampled from a colony of wild quail origin. For cross-reactive markers, polymorphism and allele frequency at each locus were estimated in 20 chickens and 20 guinea fowls made up of 10 males and 10 females randomly sampled from their respective populations. PCR products were electrophoresed on an ABI Prism 377 DNA sequencer (Perkin-Elmer, Foster City, CA) and were sized using the GENESCAN system (Perkin Elmer).

In order to confirm whether the product amplified by the cross-reactive markers was indeed the orthologous loci, 10 chicken loci and 10 guinea fowl loci were randomly selected for DNA sequencing. PCR products were purified with the High Pure PCR Product Purification Kit (Boehringer Mannheim, IN) and cycle sequence was performed using the non-labeled primer of the same primer-pair used to amplify the locus. Sequences were determined by the dye termination method employing an ABI Prism 377 DNA sequencer (Perkin 
Elmer). Sequence comparisons were made with GENETYX-Homology v.2.2.2 (Software Development, Tokyo, Japan).

\section{RESULTS}

\subsection{Fifty new Japanese quail microsatellite loci}

A total of 100 microsatellite markers were isolated and characterized. The first 50 (GUJ0001-GUJ0050) of these markers have been published elsewhere [19] while the remaining 50 markers (GUJ0051-GUJ0100) are being reported for the first time. The locus name, GenBank accession number, microsatellite repeat array, as well as primer pairs designed for these markers are shown in Table I. The number of (CA/GT $)_{n}$ repeats in the newly sequenced clones varied between 7 and 19. According to the criteria used by Weber [44], most of the new microsatellites were perfect repeats $(82.0 \%)$ and the remaining arrays were either interrupted (imperfect $6.0 \%$ ) or a compound of two perfect repeats $(12.0 \%)$. The optimized annealing temperature was from 50 to $64{ }^{\circ} \mathrm{C}$.

\subsection{Profile of Japanese quail microsatellite markers}

The characteristics of all 100 microsatellite markers based on genotyping data from 20 unrelated quails are shown in Table I. All loci (98.0\%) except GUJ0038 and GUJ0096 were polymorphic, and the average number of alleles per locus was 3.7 (range 1 to 6 alleles). The allele sizes were between 87 and $298 \mathrm{bp}$ (mean range $12.6 \mathrm{bp}$ ) and the effective number of alleles was from 1.0 to 4.3 (mean 2.45). The observed and expected heterozygosities ranged from 0.00 to 0.95 (mean 0.423 ) and 0.00 to 0.77 (mean 0.527 ), respectively. Values for the polymorphism information content $(P I C)$ varied between 0.000 and 0.729 (mean 0.4769). Based on the classification of Botstein et al. [1], 59.2\% $(58 / 98)$ of the polymorphic markers were highly informative $(P I C>0.50)$, $28.6 \%$ (28/98) were reasonably informative $(0.50>P I C>0.25)$, and $12.2 \%$ $(12 / 98)$ were slightly informative $(P I C<0.25)$.

\subsection{Cross-species amplification of Japanese quail markers in chicken and guinea fowl}

Table I also shows the results of cross-species amplification of all 100 quail markers in chicken and guinea fowl. In all, 42 loci in chicken and 20 in guinea fowl yielded analyzable PCR products that were mostly similar in size to that expected based on the fragment size of the orthologous quail loci.

The profile of the Japanese quail markers that produced positive results in the chicken is given in Table II. An average of 1.9 alleles per locus (range 1 to 4 alleles) was observed. $57.1 \%$ (24/42) of the markers were polymorphic with 
Table I. Profile of one hundred Japanese quail microsatellite markers ${ }^{\#}$.

(continued on next pages)

\begin{tabular}{|c|c|c|c|c|c|c|c|c|c|c|c|c|c|}
\hline $\begin{array}{l}\text { Locus } \\
\text { name }\end{array}$ & $\begin{array}{c}\text { GenBank } \\
\text { accession } \\
\text { number }\end{array}$ & Repeat array & Forward primer $\left(5^{\prime}-3^{\prime}\right)$ & Reverse primer $\left(5^{\prime}-3^{\prime}\right)$ & $\begin{array}{c}\text { Size } \\
\text { range } \\
\text { (bp) }\end{array}$ & $\begin{array}{c}T_{\mathrm{A}} \\
\left({ }^{\circ} \mathrm{C}\right)\end{array}$ & $N_{\mathrm{O}}$ & $N_{\mathrm{E}}$ & $H_{\mathrm{O}}$ & $H_{\mathrm{E}}$ & $P I C$ & $\begin{array}{l}\text { Amplif- } \\
\text { ication in } \\
\text { chicken }\end{array}$ & $\begin{array}{l}\text { Amplif- } \\
\text { ication in } \\
\text { guinea } \\
\text { fowl }\end{array}$ \\
\hline GUJ0001 & AB035652 & (CA)7TG(CA)13 & GAAGCGAAAGCCGAGCCA & CAGCACTTCGGAGCACAGGA & $231-239$ & 56 & 4 & 3.3 & 0.70 & 0.70 & 0.645 & + & + \\
\hline GUJ0002 & AB035813 & (CA) 13 & AGGTTGTGCTTTGCTTGTAT & GAGCATGTTGCACATTTCTT & $141-157$ & 50 & 3 & 2.0 & 0.00 & 0.51 & 0.442 & $\mathbf{0}$ & 0 \\
\hline GUJ0003 & AB035814 & (CA)9 & AGGGAAGAAGCAACTGTTC & ATTCCAGAATCTGGACTGG & $144-148$ & 48 & 2 & 1.9 & 0.50 & 0.48 & 0.365 & + & 0 \\
\hline GUJ0004 & AB037157 & (CA) 10 & AGCTCTCCTATGGGGCAAC & CTGAGCACGAGGACTGGGAA & $183-233$ & 59 & 3 & 2.5 & 0.20 & 0.60 & 0.515 & $\mathbf{0}$ & 0 \\
\hline GUJ0005 & AB035815 & (CT)11CG(CA)13 & GCTCTGCTCTCACAGCAGT & TGGATCTGGAGCTGCAACGC & $127-149$ & 59 & 4 & 3.0 & 0.30 & 0.67 & 0.620 & $\mathbf{0}$ & 0 \\
\hline GUJ0006 & AB035816 & (CA) 14 & TGGGATGATAATGAGGTACGG & AGGATAGCATTTCAGTCACGG & $117-121$ & 55 & 4 & 2.7 & 0.30 & 0.63 & 0.562 & $\mathbf{0}$ & 0 \\
\hline GUJ0007 & AB035817 & (CA) 15 & TGACTGCTTTCCACACACA & CAGAAGGTAAAAGGACGGA & $87-89$ & 51 & 2 & 1.5 & 0.25 & 0.35 & 0.288 & $\mathbf{0}$ & 0 \\
\hline GUJ0008 & AB035818 & (CA) 10 & CATGGTTATCAACCTGCAGA & ACATGCCAGTCCTTCACAAT & $170-174$ & 58 & 3 & 2.8 & 0.85 & 0.64 & 0.562 & + & 0 \\
\hline GUJ0009 & AB035819 & (CA) 14 & CACGCTTGCTTCTTGCTTCA & TATGTTTGGTGCCCTGCTAG & $199-203$ & 60 & 2 & 1.2 & 0.20 & 0.18 & 0.164 & $\mathbf{0}$ & 0 \\
\hline GUJ0010 & AB035820 & (CA) 15 & TTCCTTCTGGGTGCTGCTCA & САTAGACACATCССТСССТC & $154-158$ & 62 & 2 & 1.5 & 0.35 & 0.35 & 0.288 & + & 0 \\
\hline GUJ0011 & AB035821 & (CA) 13 & TACTTGATACACCAGCTGTC & САСССТАТАССАATGAAAGG & $159-167$ & 58 & 4 & 2.3 & 0.24 & 0.56 & 0.469 & $\mathbf{0}$ & 0 \\
\hline GUJ0012 & AB035822 & (CA)6TA(CA)6 & TTTATGTACTGTTTGGGCGC & CTTGGACATAGAGTAAGCCA & $140-146$ & 58 & 3 & 2.7 & 0.35 & 0.63 & 0.555 & $\mathbf{0}$ & 0 \\
\hline GUJ0013 & AB035823 & (CA) 10 & ACCAAACCCGAGATCCGACA & AGCGTTCGCGTTCCTCTTTC & $127-139$ & 55 & 4 & 3.0 & 0.75 & 0.67 & 0.611 & + & + \\
\hline GUJ0014 & AB035824 & (CA) 9 & TGCTGGGGTTGCTTTCTCCA & TCTCGGTGGTTTGCTCTGAC & $143-147$ & 60 & 3 & 1.7 & 0.45 & 0.41 & 0.345 & + & 0 \\
\hline GUJ0015 & AB035825 & (CA) 9 & AGGTGGTCCCCAATGCCCTT & GGAAGCAGAGCATCGTTCCC & $135-139$ & 60 & 2 & 1.2 & 0.05 & 0.14 & 0.130 & $\mathbf{0}$ & 0 \\
\hline GUJ0016 & AB035826 & (CA) 9 & AATGAATGTCTGGGTGGTGC & CATGGAGTGTTGGGTATTGC & $235-249$ & 55 & 2 & 1.1 & 0.00 & 0.10 & 0.090 & $\mathbf{0}$ & 0 \\
\hline GUJ0017 & AB035827 & (CA) 14 & AGAGAGATTAGAGGAGCTGC & GGCACTAAAACCATCGAGAG & $153-165$ & 60 & 2 & 1.9 & 0.30 & 0.48 & 0.365 & + & + \\
\hline GUJ0018 & AB035828 & (CA)10 & ATCCCGCGCCGTCCTTTGTT & CGGCACCACGAAGTACTCCA & $237-243$ & 55 & 2 & 1.8 & 0.30 & 0.46 & 0.351 & + & 0 \\
\hline GUJ0019 & AB035829 & (CA) 21 & GGGGGCTGTAGGTCTGGATC & ATCGGGCACC & 183-191 & 50 & 4 & 2.4 & 0.40 & 0.58 & 0.495 & $\mathbf{0}$ & 0 \\
\hline GUJ0020 & AB035830 & (CA) 8 & AATGTCCTTGTGCAGCTCCA & CAGCATTGTGCAAAGCAGTG & 205-207 & 64 & 2 & 1.2 & 0.00 & 0.18 & 0.164 & $\mathbf{0}$ & 0 \\
\hline GUJ0021 & AB035831 & (CA) 11 & GAGCATTTCTAGTCTGTCTC & GATCAATACACAGGCTAAGG & $143-157$ & 62 & 4 & 3.9 & 0.65 & 0.74 & 0.696 & + & + \\
\hline GUJ0022 & AB035832 & (CA) 15 & AAACTTATTCTCGCGCTCCC & TAAGCAAGGAAGAGGTGGCA & $126-132$ & 69 & 3 & 2.1 & 0.95 & 0.52 & 0.409 & $\mathbf{0}$ & 0 \\
\hline GUJ0023 & AB035833 & (CA)7TA( & GAGAGGTACAGCAACACTTT & CGTTTCTTTCTGGAGTGTCT & $219-237$ & 55 & 4 & 2.6 & 0.40 & 0.61 & 0.545 & + & + \\
\hline GUJ0024 & AB035834 & (CA) 13AA(CA)3 & TCACACCTTCGGGCTGATCT & ATGCGACGGGGTGCCTTAAA & $162-174$ & 55 & 6 & 4.3 & 0.80 & 0.77 & 0.725 & $\mathbf{0}$ & 0 \\
\hline GUJ0025 & AB035835 & (CA) 9 & CCTGAGCGAATACACAACTG & AGTGTTAGGTGAGGACTGCT & $243-247$ & 60 & 2 & 2.0 & 0.35 & 0.50 & 0.374 & $\mathbf{0}$ & 0 \\
\hline GUJ0026 & AB035836 & (CA) 16 & CATGAACATCTCTCTTCATG & GTGTTCTGCATCACAAACAT & $112-118$ & 60 & 2 & 1.1 & 0.00 & 0.10 & 0.090 & $\mathbf{0}$ & 0 \\
\hline GUJ0027 & AB035837 & (CA) 15 & TTCACAGATGACAATCTAGC & CTGCAAGTAACAGAAGGTAA & $163-177$ & 55 & 4 & 1.6 & 0.40 & 0.38 & 0.359 & + & 0 \\
\hline GUJ0028 & AB035838 & (CA) 9 & TGAACAAAGCAGAAAGGAGC & CСТTACCTACATGAAACGTC & $150-178$ & 55 & 5 & 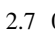 & 0.55 & 0.63 & 0.579 & 0 & 0 \\
\hline GUJ0029 & AB035839 & (СА)11CT(CA)2 & GAGCATTTCTAGTCTGTCTC & ATACACAGGCTAAGGAAACC & $140-152$ & 55 & 5 & 2.9 & 0.80 & 0.66 & 0.598 & + & + \\
\hline GUJ0030 & AB035840 & (CA)31 & TGCACCAATCCCAGCTGTTT & AACGCACAATGGAAAGTGGG & $167-179$ & 64 & 5 & 4.2 & 0.35 & 0.76 & 0.727 & $\mathbf{0}$ & 0 \\
\hline GUJ0031 & AB035841 & (CA) 9 & AAGGGCAGGGGCTGGGAACA & CGCCTCTGCGGTGTGCAACT & $160-166$ & 55 & 4 & 3.1 & 0.45 & 0.68 & 0.612 & + & 0 \\
\hline GUJ0032 & AB035842 & (CA) 5CTG(CA)9 & GAGGCTGCGAACAACACACA & GCTAAGACGAGGTGAAGGCT & 161-197 & 55 & 3 & 1.6 & 0.25 & 0.36 & 0.310 & o & 0 \\
\hline
\end{tabular}


Table I. Continued.

\begin{tabular}{|c|c|c|c|c|c|c|c|c|c|c|c|c|}
\hline $\begin{array}{l}\text { Locus } \\
\text { name }\end{array}$ & $\begin{array}{c}\text { GenBank } \\
\text { accession } \\
\text { number }\end{array}$ & Repeat array & Forward primer $\left(5^{\prime}-3^{\prime}\right)$ & Reverse primer $\left(5^{\prime}-3^{\prime}\right)$ & $\begin{array}{l}\text { Size } \\
\text { range } \\
\text { (bp) }\end{array}$ & $\begin{array}{c}T_{\mathrm{A}} \\
\left({ }^{\circ} \mathrm{C}\right)\end{array}$ & $N_{\mathrm{O}}$ & $N_{\mathrm{E}} H_{\mathrm{O}}$ & $H_{\mathrm{E}}$ & $P I C$ & $\begin{array}{l}\text { Amplif- } \\
\text { ication in } \\
\text { chicken }\end{array}$ & $\begin{array}{l}\text { Amplif- } \\
\text { ication in } \\
\text { guinea } \\
\text { fowl }\end{array}$ \\
\hline GUJ0033 & AB035843 & (CA)13 & TCTGCTCTCACAGCAGTGCA & GCATAGAGCCCAGCAGTGTT & $193-203$ & 55 & 5 & 2.10 .45 & 0.51 & 0.483 & $\mathbf{0}$ & 0 \\
\hline GUJ0034 & AB035844 & (CA)9CG(CA)2 & CGTAACGGTCCAATATGGAT & TCCACGATGCAGAGGTATTT & $219-241$ & 55 & 5 & 4.20 .60 & 0.76 & 0.727 & + & 0 \\
\hline GUJ0035 & AB035845 & (CA) 14 & AATACTGGTTTTGTGATGGC & GGGCAATAAAAGAAAGACTG & $144-150$ & 55 & 3 & 2.60 .75 & 0.61 & 0.539 & $\mathbf{0}$ & 0 \\
\hline GUJ0036 & AB035846 & (CA)9TA(CA)4 & CTTTCACATTGCTTTTGCCT & CACTAAAGATTGGCTAACAG & $147-155$ & 55 & 4 & 1.40 .10 & 0.27 & 0.250 & $\mathbf{0}$ & 0 \\
\hline GUJ0037 & AB035847 & (CA) $10 \mathrm{C}(\mathrm{CA}) 2$ & CCATTCCTCCATCGTTCTGA & GGGAAGGAGTGTAGGAAAGA & 178-194 & 55 & 4 & 1.90 .30 & 0.48 & 0.448 & $\mathbf{0}$ & 0 \\
\hline GUJ0038 & AB035848 & (CA)19 & TACATCCAGCAATCGCCCAC & CACGGGTGAGTCCATTAGTG & 262 & 60 & 1 & 1.00 .00 & 0.00 & 0.000 & $\mathbf{0}$ & 0 \\
\hline GUJ0039 & AB035849 & (CA)19 & CAAAGAGCAGAGGGAATGGA & CCGAGAGATGGGTTTTTTCC & $164-188$ & 60 & 4 & 3.40 .70 & 0.71 & 0.659 & $\mathbf{0}$ & + \\
\hline GUJ0040 & AB035850 & (CA) 12 & GTTGAAGCTCCCATCCCTCC & ACACCCCCACGGTCTTTGCA & 176-192 & 55 & 4 & 2.30 .20 & 0.56 & 0.494 & $\mathbf{0}$ & + \\
\hline GUJ0041 & AB035851 & (CA) 11 & AAAATGTCTGCAAAATGGGC & TGAAACATACCTGAGTGCTA & $114-126$ & 55 & 4 & 3.90 .45 & 0.75 & 0.697 & $\mathbf{0}$ & 0 \\
\hline GUJ0042 & AB035852 & (CA) 8 & TCAGTGCCTTTGTGTTGTCC & ACAGCCTTCCCCAAATTCCT & 189-191 & 55 & 2 & 1.30 .00 & 0.26 & 0.222 & + & 0 \\
\hline GUJ0043 & AB035853 & (CA)9TGTG(CA)2 & GAGACCAGGTGGTCCCCAAT & GGAAGCAGAGCATCGTTCCC & $141-145$ & 55 & 2 & 1.20 .00 & 0.18 & 0.164 & 0 & 0 \\
\hline GUJ0044 & AB035854 & (CA)16 & GCCTTGAAACCTGAGTGATC & TGCATTTCAGCAGCTCTCAG & $180-220$ & 55 & 5 & 3.50 .75 & 0.72 & 0.666 & + & 0 \\
\hline GUJ0045 & AB035855 & (CA) 18 & ACATGCACCACCATTCTTGC & CATGCACAAATGAGCGTGCA & $241-251$ & 60 & 2 & 1.10 .05 & 0.05 & 0.048 & $\mathbf{0}$ & 0 \\
\hline GUJ0046 & AB035856 & (CA) 9 & GCCATGTTTGTCACCTTGCA & ACTGGTTGGGACTGAAGGAT & $206-210$ & 55 & 3 & 2.20 .35 & 0.54 & 0.481 & + & 0 \\
\hline GUJ0047 & AB035857 & (CA) 23 & GAGATAAGACTGGCTGGGGC & TCACCGTGGCTGGCCAACTT & $262-292$ & 55 & 5 & 2.40 .55 & 0.59 & 0.555 & + & 0 \\
\hline GUJ0048 & AB035858 & (CA) 14 & AACGCATACAACTGACTGGG & GGATAGCATTTCAGTCACGG & $130-138$ & 55 & 4 & 3.80 .85 & 0.74 & 0.688 & $\mathbf{0}$ & 0 \\
\hline GUJ0049 & AB035859 & (CA)11 & GAAGCAGTGACAGCAGAATG & CGGTAGCATTTCTGACTCCA & $229-241$ & 55 & 5 & 4.20 .75 & 0.76 & 0.725 & + & 0 \\
\hline GUJ0050 & AB035860 & (CA) 8 & CTGCCATGTTACTAATCTAG & TGGTTTCTTTACACTTGACA & $143-153$ & 55 & 3 & 1.10 .10 & 0.10 & 0.094 & + & 0 \\
\hline GUJ0051 & AB063119 & (CA) 10 & ССТТААССАСТССТАСТGАC & TTTTGTAAGTGGCCCCGTAC & $184-188$ & 55 & 2 & 1.10 .00 & 0.10 & 0.090 & 0 & 0 \\
\hline GUJ0052 & AB063120 & (CA) 12 & AAACTACCGATGTAAGTAAG & ATGAGATATATAAGGAACCC & $96-108$ & 55 & 5 & 3.70 .55 & 0.73 & 0.681 & 0 & 0 \\
\hline GUJ0053 & AB063121 & (CA) 19 & GCTGGAGTTTTACATGCACG & TGGATTATGATGCTGACATAAG & $151-159$ & 64 & 4 & 3.00 .60 & 0.67 & 0.608 & 0 & 0 \\
\hline GUJ0054 & AB063122 & (CA)7 & GTGTTCTCTCACTCCCCAAT & ATGTGAGCAATTGGGACTG & $120-146$ & 55 & 4 & 2.70 .55 & 0.63 & 0.569 & + & 0 \\
\hline GUJ0055 & AB063123 & (CT)12(CA)11 & GCATACTGCAATATACCTGA & TTGACATACTTGGATTAGAGA & $159-183$ & 55 & 5 & 2.50 .20 & 0.59 & 0.540 & 0 & 0 \\
\hline GUJ0056 & AB063124 & (CA)7 & GTTACATCCATCCTGCCTCA & CTCTTGAGCCTACCAGTCTG & $181-185$ & 55 & 3 & 2.70 .15 & 0.63 & 0.532 & + & 0 \\
\hline GUJ0057 & AB063125 & (CA) 12 & GGAATGGAAAATATGAGAGC & CAGGTGTTAAAGTCCAATGT & $132-154$ & 62 & 5 & 2.40 .65 & 0.59 & 0.544 & + & 0 \\
\hline GUJ0058 & AB063126 & (CA) 10 & CCCTTCCAAGTTCCTGG & ATGACAGGTCCAGCCTG & 103-109 & 55 & 4 & 3.10 .35 & 0.67 & 0.598 & + & 0 \\
\hline GUJ0059 & AB063127 & (CA) 10 & GACAAAGTTACAGCTAGGAG & TAGGTGCGAAAATCTCTGAC & 207-219 & 50 & 5 & 3.40 .85 & 0.71 & 0.670 & + & + \\
\hline GUJ0060 & AB063128 & (CA) 9 & ATGCTATGGGAACCTCACTC & TATAAAGCAGGGGGACATGG & $132-168$ & 60 & 5 & 1.60 .40 & 0.38 & 0.357 & 0 & 0 \\
\hline GUJ0061 & AB063129 & (CA) 15 & ССАСGСТССССААТTТССТG & CCTTGGAGTGCTTCCAAGCG & $157-171$ & 55 & 5 & 3.20 .60 & 0.69 & 0.620 & + & + \\
\hline GUJ0062 & AB063130 & (CA) 13 & TTATGTTTGATGGGCAGAGG & CATGGCAAAAACTGAAGAGC & $171-201$ & 60 & 4 & 1.50 .40 & 0.35 & 0.329 & 0 & 0 \\
\hline GUJ0063 & AB063131 & (CA) $7 \mathrm{CT}(\mathrm{CA}) 2 \mathrm{CT}(\mathrm{CA}) 7$ & GCTCAGGTTCTCAGCTGATG & GGGAGAGATCAAGGGAACAG & $242-250$ & 55 & 4 & 2.50 .60 & 0.61 & 0.538 & + & + \\
\hline GUJ0064 & AB063132 & (CA) 8 & AAGCCTGATTCCCTGCCTTG & TTAAAGCTGGGAGGTGGAGG & $214-220$ & 55 & 4 & 1.60 .20 & 0.38 & 0.351 & 0 & + \\
\hline
\end{tabular}


Table I. Continued.

\begin{tabular}{|c|c|c|c|c|c|c|c|c|c|c|c|c|c|}
\hline $\begin{array}{l}\text { Locus } \\
\text { name }\end{array}$ & $\begin{array}{c}\text { GenBank } \\
\text { accession } \\
\text { number }\end{array}$ & Repeat array & Forward primer $\left(5^{\prime}-3^{\prime}\right)$ & Reverse primer $\left(5^{\prime}-3^{\prime}\right)$ & $\begin{array}{c}\text { Size } \\
\text { range } \\
\text { (bp) }\end{array}$ & $\begin{array}{c}T_{\mathrm{A}} \\
\left({ }^{\circ} \mathrm{C}\right)\end{array}$ & $N_{\mathrm{O}}$ & $N_{\mathrm{E}}$ & $H_{\mathrm{O}}$ & $H_{\mathrm{E}}$ & $P I C$ & $\begin{array}{c}\text { Amplif- } \\
\text { ication in } \\
\text { chicken }\end{array}$ & $\begin{array}{l}\text { Amplif- } \\
\text { ication in } \\
\text { guinea } \\
\text { fowl }\end{array}$ \\
\hline GUJ0065 & AB063133 & (CA) 13 & GCGTGCCATTTACTTCCCGG & AGCCAGGATGACCAGGAAGG & $109-131$ & 55 & 5 & 2.3 & 0.55 & 0.57 & 0.536 & + & 0 \\
\hline GUJ0066 & AB063134 & (CA) $12 \mathrm{TA}(\mathrm{CA}) 2$ & GGGAAAACAATCACTGCCTC & TCTGCAAATCCCCCTTAGAG & $167-175$ & 55 & 3 & 1.1 & 0.10 & 0.10 & 0.094 & + & + \\
\hline GUJ0067 & AB063135 & (CA) 14 & ACGTACGAGCTCAACATTTG & GCGTGCATAAAGGCAACTTA & $121-131$ & 55 & 5 & 2.8 & 0.85 & 0.65 & 0.594 & 0 & 0 \\
\hline GUJ0068 & АВ063136 & (CA) 13 & TAGGAGAGGTCACGATTTGC & ATCTTAACTCGCCCAGCCTT & 204-216 & 54 & 5 & 3.6 & 0.60 & 0.72 & 0.668 & 0 & 0 \\
\hline GUJ0069 & AB063137 & (CA) 11 & TTCAGGGTAGCAGTCATCTC & CACCAACCACCTTCATCTTC & 201-211 & 54 & 2 & 1.7 & 0.40 & 0.42 & 0.332 & 0 & 0 \\
\hline GUJ0070 & AB063138 & $(\mathrm{CA}) 9$ & AAACCCCAAAGAAGCTGTCC & ACGTTGTCACCATCAGCTTG & 196-206 & 54 & 6 & 4.3 & 0.62 & 0.77 & 0.729 & + & 0 \\
\hline GUJ0071 & AB063139 & (CA) 8 & AGATCCTGCTCCTGGAATTG & CAGCTGCACTTAATACAGGC & $160-178$ & 54 & 6 & 2.0 & 0.30 & 0.49 & 0.468 & 0 & 0 \\
\hline GUJ0072 & AB063140 & (CA) 13 & СTTTCTTTCTGGCATTGTAC & ATGGGAAGTTGTAGTAGTAG & $114-120$ & 50 & 3 & 1.6 & 0.50 & 0.39 & 0.618 & 0 & 0 \\
\hline GUJ0073 & AB063141 & (CA) 13 & GCTGCTATTCTGTTGATGTG & CAACTGCAAAGACAACATCC & $144-160$ & 52 & 4 & 3.1 & 0.55 & 0.68 & 0.618 & 0 & + \\
\hline GUJ0074 & AB063142 & (CA) 10 & GTTGTCCTGGCTGAGATGGC & GGGTTTGAGGGCTTGGGGTT & $290-298$ & 59 & 3 & 2.2 & 0.60 & 0.54 & 0.455 & 0 & 0 \\
\hline GUJ0075 & AB063143 & (CA) 8 & CTCCAATCACACTAGCTCTG & CCTGCTTTTTTTGGGAGAGG & $122-126$ & 54 & 2 & 1.2 & 0.15 & 0.14 & 0.121 & 0 & 0 \\
\hline GUJ0076 & AB063144 & (CA)4AA(CA) 9 & GTATCAGTGCATGCTCGTCC & TCGAGGACTGGCTGGAAAAT & $208-230$ & 57 & 5 & 2.3 & 0.80 & 0.57 & 0.494 & 0 & 0 \\
\hline GUJ0077 & AB063145 & (CA) 8 & TATAAGATGGGGAGTGGCAG & ATTTTGCTGACCCCCTTCTG & $228-232$ & 54 & 4 & 2.1 & 0.60 & 0.52 & 0.443 & + & 0 \\
\hline GUJ0078 & АВ063146 & (CA) 14 & TCTTTGATTGATGGCTTGCG & GTTATCCTCTGAAGTGTAGC & $141-149$ & 55 & 4 & 2.2 & 0.30 & 0.55 & 0.495 & 0 & 0 \\
\hline GUJ0079 & AB063147 & (CA) 12 & GAAAGATAAGCATGAGTGAC & GTTTTGGCATTCACTTCAGA & $121-135$ & 55 & 6 & 3.0 & 0.65 & 0.67 & 0.626 & 0 & 0 \\
\hline GUJ0080 & АВ063148 & (CA)9 & TTGAAGGGACATAGGGAAGC & GAAAACGGTGAAGTCTGGTG & $151-167$ & 54 & 6 & 4.2 & 0.35 & 0.76 & 0.728 & 0 & 0 \\
\hline GUJ0081 & AB063149 & (CA) 14 & AGGAACGAGTGGAAGTGAAG & TTGGAAAGACACGTTGGGCT & $134-144$ & 54 & 3 & 2.4 & 0.65 & 0.59 & 0.506 & 0 & 0 \\
\hline GUJ0082 & АВ063150 & (CA)9 & CTTGGAACACACGGGATGGC & ТТАССССТСТТТТСССССCG & $142-156$ & 59 & 5 & 2.7 & 0.30 & 0.63 & 0.558 & + & 0 \\
\hline GUJ0083 & АB063151 & (CA) 11 & CCATCTCTGTGCCTTTCCAA & GCTGAAAACATTGGGCGTAG & $118-128$ & 55 & 3 & 2.8 & 0.45 & 0.64 & 0.567 & 0 & 0 \\
\hline GUJ0084 & AB063152 & (CA) 10 & АСТССТССТСТТТСТСССТС & TCCCGTCTCCCGATGTGTTT & $159-165$ & 55 & 3 & 2.6 & 0.55 & 0.61 & 0.531 & + & + \\
\hline GUJ0085 & AB063153 & (GT) 14 & ACAACCACTTCTCCAGCTAC & GCTTGTGCTGCTGTTGCTAA & $245-265$ & 55 & 5 & 2.4 & 0.65 & 0.59 & 0.548 & + & 0 \\
\hline GUJ0086 & AB063154 & (CA) 19 & AGCTGCCATATCTACTGCTC & TGGCTTAGTGCTTTCAGAGG & $197-207$ & 55 & 4 & 3.8 & 0.40 & 0.73 & 0.684 & + & + \\
\hline GUJ0087 & AB063155 & (CT)12AA(CA) 11 & CATGCCGGCTGCTATGACAG & AAGTGCAGGGAGCGAGGAAG & $151-155$ & 55 & 3 & 2.8 & 0.65 & 0.65 & 0.572 & + & + \\
\hline GUJ0088 & AB063156 & (CA) 21 & TCTTCACCCTCACTGTATGC & ATCCACGTACAAAGCGTTGC & $165-189$ & 55 & 3 & 2.6 & 0.11 & 0.61 & 0.542 & 0 & 0 \\
\hline GUJ0089 & AB063157 & (CA) 12 & CCAGTTTAAGCACCAGCATC & TGGCAAGTAGTCGTGGAAGA & $131-145$ & 55 & 5 & 2.5 & 0.79 & 0.60 & 0.524 & 0 & + \\
\hline GUJ0090 & AB063158 & (CA) $11(\mathrm{TA}) 4$ & GCCTTCAGAGTGGGAAAT & TCTCACAGAAACAGCTCC & $96-106$ & 55 & 4 & 2.9 & 0.20 & 0.66 & 0.588 & 0 & 0 \\
\hline
\end{tabular}


Table I. Continued.

\begin{tabular}{|c|c|c|c|c|c|c|c|c|c|c|c|c|c|}
\hline $\begin{array}{l}\text { Locus } \\
\text { name }\end{array}$ & $\begin{array}{c}\text { GenBank } \\
\text { accession } \\
\text { number }\end{array}$ & Repeat array & Forward primer $\left(5^{\prime}-3^{\prime}\right)$ & Reverse primer $\left(5^{\prime}-3^{\prime}\right)$ & $\begin{array}{c}\text { Size } \\
\text { range } \\
\text { (bp) }\end{array}$ & $\begin{array}{c}T_{\mathrm{A}} \\
\left({ }^{\circ} \mathrm{C}\right)\end{array}$ & $N_{\mathrm{O}}$ & $N_{\mathrm{E}}$ & $H_{\mathrm{O}}$ & $H_{\mathrm{E}}$ & $P I C$ & $\begin{array}{l}\text { Amplif- } \\
\text { ication in } \\
\text { chicken }\end{array}$ & $\begin{array}{c}\text { Amplif- } \\
\text { ication in } \\
\text { guinea } \\
\text { fowl }\end{array}$ \\
\hline GUJ0091 & AB063159 & (CA) 9 & AAACCGCCATCCCCATTCC & AGCACGTGGGCAAAGGAAC & $172-188$ & 55 & 3 & 2.7 & 0.70 & 0.63 & 0.645 & + & + \\
\hline GUJ0092 & AB063160 & (TA) $7(\mathrm{CA}) 12$ & GTACATTGCTTGCCAGTA & TCCAAGTATGTTGCTTGC & $117-123$ & 55 & 4 & 3.0 & 0.55 & 0.66 & 0.599 & 0 & 0 \\
\hline GUJ0093 & AB063161 & (CA) 16 & CTCTTGTATTGTAACTGGGC & AGCCATAGAGGGCTATTAAG & 213-231 & 60 & 4 & 3.1 & 0.45 & 0.68 & 0.612 & + & 0 \\
\hline GUJ0094 & AB063162 & (CA) 16 & ATTTTCCCCTCCTTGTCATG & CACTGTTCACTGTTATTCCC & 237-249 & 55 & 4 & 2.3 & 0.15 & 0.56 & 0.522 & + & + \\
\hline GUJ0095 & AB063163 & (CA) 12 & GCAACATTTTCAGTCAGATC & AАTTCTCATCAGTCTCCAAC & $120-126$ & 55 & 2 & 1.4 & 0.37 & 0.30 & 0.255 & 0 & 0 \\
\hline GUJ0096 & AB063164 & (A) $10(\mathrm{CA}) 14(\mathrm{~A}) 20$ & GTACCAAAAGTGAATAGTGG & CAGATCACAGACTTAGAAAG & 157 & 55 & 1 & 1.0 & 0.00 & 0.00 & 0.000 & 0 & 0 \\
\hline GUJ0097 & AB063165 & (CA) 14 & GGATGCTCAGTGTGGAAAAG & GAGCAAGAGGTGAGTGTTTC & $131-157$ & 55 & 5 & 3.6 & 0.40 & 0.72 & 0.672 & + & 0 \\
\hline GUJ0098 & AB063166 & (CA) 12 & GCATAACTGAACTACCACGC & GCATCAGTTCCATCAGCTAG & $197-205$ & 55 & 4 & 2.5 & 0.73 & 0.60 & 0.539 & + & 0 \\
\hline GUJ0099 & AB063167 & (CA)16GA(CA)5(TA)7 & СТСТТАТССАТССТТССТТС & TTTTAAGTTTCCCCAGGCAG & $246-284$ & 55 & 3 & 3.0 & 0.30 & 0.66 & 0.590 & + & 0 \\
\hline GUJ0100 & AB063168 & (CA) 12 & GCATTTCCATCAGTACAACC & CAGAATATAAGGTCACAGCC & $278-290$ & 55 & 5 & 2.8 & 0.45 & 0.65 & 0.602 & 0 & 0 \\
\hline
\end{tabular}

\# The locus code GUJ stands for Gifu University Japanese quail and is in accordance with the standardized nomenclature rules adopted for poultry [5]. $T_{\mathrm{A}}$, annealing temperature; $N_{\mathrm{O}}$ observed number of alleles; $N_{\mathrm{E}}$, effective number of alleles; $H_{\mathrm{O}}$, observed heterozygosity; $H_{\mathrm{E}}$, expected heterozygosity; $P I C$, polymorphism information content; + , amplification products were obtained using the annealing temperature optimized for quails; 0 , amplification products were not obtained using the annealing temperature optmized for quails.

The information provided in bold type for the first 50 markers, GUJ0001-GUJ0050, has been originally published in The Journal of Heredity [19]. 
2 to 4 alleles per locus and $42.9 \%$ (18/42) were monomorphic. The observed heterozygosity and $P I C$ were on average 0.205 and 0.1888 , respectively. Based on the PIC, $12.5 \%(3 / 24)$ of the polymorphic markers were highly informative, $58.3 \%$ (14/24) reasonably informative, and $29.2 \%$ (7/24) slightly informative. Nearly $60.0 \%$ (25/42) of the markers amplified chicken loci at $1.5 \mathrm{mM} \mathrm{MgCl} 2$ concentration, which is the same as that used in amplifying quail loci. However, the $\mathrm{MgCl}_{2}$ concentration had to be adjusted to $2.0 \mathrm{mM}$ for 15 markers and $2.5 \mathrm{mM}$ for the GUJ0018 and GUJ0098 markers.

The characteristics of the Japanese quail microsatellite loci that were amplified in guinea fowl are shown in Table III. The observed number of alleles per locus averaged 1.9 (range 1 to 5 alleles). A polymorphism was observed in $55.0 \%(11 / 20)$ of the markers having 2 to 5 alleles per locus, while the rest were monomorphic. The mean observed heterozygosity was 0.127 and that of PIC was 0.1553 . Of the polymorphic markers, $18.2 \%(2 / 11)$ were highly informative, $36.4 \%$ (4/11) were reasonably informative, and $45.5 \%(5 / 11)$ were slightly informative. Similar to chicken, $70.0 \%(14 / 20)$ of the markers amplified guinea fowl loci at $1.5 \mathrm{mM} \mathrm{MgCl}_{2}$ concentration, with four markers requiring $2.0 \mathrm{mM} \mathrm{MgCl}_{2}$ and two markers (GUJ0089 and GUJ0091) requiring $2.5 \mathrm{mM}$ of $\mathrm{MgCl}_{2}$.

\subsection{Japanese quail, chicken and guinea fowl loci amplified by the same quail markers}

Fifteen Japanese quail markers were found to cross-amplify both chicken and guinea fowl DNA. To illustrate how informative these markers would be for comparative mapping, their observed heterozygosities were plotted in Figure 1. Generally, nearly all the 15 loci had high heterozygosities in Japanese quail, which is not unexpected since they are quail-specific markers. Five loci in chicken (GUJ0059, GUJ0061, GUJ0066, GUJ0087, and GUJ0094) and 7 loci in guinea fowl (GUJ0001, GUJ0013, GUJ0021, GUJ0029, GUJ0061, GUJ0087, and GUJ0091) were not heterozygous and therefore uninformative in our test populations. However, 5 loci (GUJ0017, GUJ0023, GUJ0063, GUJ0084, and GUJ0086) were informative in all three species of Phasianidae and would thus be useful for comparative mapping. The average observed heterozygosities for these 15 loci in the Japanese quail, chicken and guinea fowl were $0.547,0.297$, and 0.145 , respectively.

\subsection{Sequence analysis of chicken and guinea fowl loci amplified by Japanese quail markers}

The sequence information of 10 chicken loci amplified by cross-species PCR is summarized in Table IV. Nine chicken loci contained (CA/GT) repeats, 5 (GUC0002, GUC0003, GUC0006, GUC0007, and GUC0009) of 
Table II. Characteristics of 42 Japanese quail microsatellite loci amplified in chicken \#

\begin{tabular}{|c|c|c|c|c|c|c|c|c|c|}
\hline $\begin{array}{l}\text { Locus } \\
\text { name }\end{array}$ & $\begin{array}{l}\text { Size range } \\
\text { (bp) } \\
\text { in quail }\end{array}$ & $\begin{array}{c}T_{\mathrm{A}} \\
\left({ }^{\circ} \mathrm{C}\right)\end{array}$ & $\begin{array}{c}\left.\mathrm{MgCl}_{2}\right] \\
(\mathrm{mM})\end{array}$ & $\begin{array}{l}\text { Size range } \\
\text { (bp) } \\
\text { in chicken }\end{array}$ & $N_{\mathrm{O}}$ & $N_{\mathrm{E}}$ & $H_{\mathrm{O}}$ & $H_{\mathrm{E}}$ & $P I C$ \\
\hline GUJ0001* & $231-239$ & 56 & 1.5 & $225-247$ & 4 & 2.3 & 0.40 & 0.56 & 0.516 \\
\hline GUJ0003 & $144-148$ & 48 & 1.5 & 134 & 1 & 1.0 & 0.00 & 0.00 & 0.000 \\
\hline GUJ0008 & $170-174$ & 58 & 1.5 & 168 & 1 & 1.0 & 0.00 & 0.00 & 0.000 \\
\hline GUJ0010 & $154-158$ & 62 & 1.5 & 160 & 1 & 1.0 & 0.00 & 0.00 & 0.000 \\
\hline GUJ0013* & $127-139$ & 55 & 1.5 & $140-144$ & 3 & 2.4 & 0.35 & 0.58 & 0.494 \\
\hline GUJ0014 & $143-147$ & 60 & 2.0 & $159-163$ & 2 & 1.1 & 0.05 & 0.05 & 0.048 \\
\hline GUJ0017* & $153-165$ & 60 & 1.5 & $149-151$ & 2 & 1.2 & 0.20 & 0.18 & 0.164 \\
\hline GUJ0018 & $237-243$ & 55 & 2.5 & 231 & 1 & 1.0 & 0.00 & 0.00 & 0.000 \\
\hline GUJ0021 & $143-1$ & 62 & 1.5 & $137-141$ & 2 & 1.1 & 0.10 & 0.10 & 0.090 \\
\hline GUJ0023* & $219-237$ & 55 & 1.5 & $208-222$ & 3 & 1.7 & 0.40 & 0.41 & 0.368 \\
\hline GUJ0027 & $163-177$ & 55 & 1.5 & $167-169$ & 2 & 1.8 & 0.65 & 0.44 & 0.343 \\
\hline GUJ0029* & $140-152$ & 55 & 1.5 & $132-$ & 2 & 1.1 & 0.10 & 0.10 & 0.090 \\
\hline GUJ0031 & $160-166$ & 55 & 2.0 & 212 & 1 & 1.0 & 0.00 & 0.00 & 0.000 \\
\hline GUJ0034 & $219-241$ & 55 & 2.0 & 163 & 1 & 1.0 & 0.00 & 0.00 & 0.000 \\
\hline GUJ0042* & 189-191 & 55 & 1.5 & 199 & 1 & 1.0 & 0.00 & 0.00 & 0.000 \\
\hline GUJ0044* & $180-220$ & 55 & 1.5 & 187 & 1 & 1.0 & 0.00 & 0.00 & 0.000 \\
\hline GUJ0046 & $206-210$ & 55 & 1.5 & $227-229$ & 2 & 1.1 & 0.05 & 0.50 & 0.048 \\
\hline GUJ0047 & $262-292$ & 55 & 2.0 & $225-233$ & 2 & 2.0 & 0.25 & 0.50 & 0.374 \\
\hline GUJ0049* & $229-241$ & 55 & 1.5 & 239 & 3 & 1.8 & 0.35 & 0.43 & 0.390 \\
\hline GUJ0050 & $143-153$ & 55 & 2.0 & 147 & 1 & 1.0 & 0.00 & 0.00 & 0.000 \\
\hline GUJ0054 & $120-146$ & 55 & 2.0 & 127 & 1 & 1.0 & 0.00 & 0.00 & 0.000 \\
\hline GUJ0056 & $181-185$ & 55 & 2.0 & 180 & 1 & 1.0 & 0.00 & 0.00 & 0.000 \\
\hline GUJ0057 & $132-154$ & 62 & 1.5 & $120-$ & 4 & 1.9 & 0.15 & 0.47 & 0.433 \\
\hline GUJ0058 & 103-109 & 55 & 2.0 & $97-99$ & 2 & 2.0 & 0.67 & 0.49 & 0.369 \\
\hline GUJ0059* & $207-219$ & 50 & 1.5 & $196-216$ & 2 & 1.8 & 0.00 & 0.45 & 0.351 \\
\hline GUJ0061 & $157-171$ & 55 & 1.5 & 158 & 1 & 1.0 & 0.00 & 0.00 & 0.000 \\
\hline GUJ0063* & $242-250$ & 55 & 1.5 & $231-235$ & 2 & 1.8 & 0.65 & 0.44 & 0.343 \\
\hline GUJ0065 & $109-131$ & 55 & 1.5 & $112-126$ & 3 & 1.6 & 0.15 & 0.39 & 0.329 \\
\hline GUJ0066 & $167-175$ & 55 & 2.0 & 176 & 1 & 1.0 & 0.00 & 0.00 & 0.000 \\
\hline GUJ0070 & $196-206$ & 54 & 2.0 & 200-204 & 2 & 1.7 & 0.55 & 0.40 & 0.319 \\
\hline GUJ0077 & $228-232$ & 54 & 2.0 & 214 & 1 & 1.0 & 0.00 & 0.00 & 0.000 \\
\hline GUJ0082 & $142-156$ & 59 & 2.0 & 140 & 1 & 1.0 & 0.00 & 0.00 & 0.000 \\
\hline GUJ0084 & $159-165$ & 55 & 1.5 & $164-176$ & 4 & 3.6 & 0.95 & 0.72 & 0.671 \\
\hline GUJ0085 & $245-265$ & 55 & 2.0 & 225 & 1 & 1.0 & 0.00 & 0.00 & 0.000 \\
\hline GUJ0086 & $197-207$ & 55 & 1.5 & $209-215$ & 3 & 2.7 & 1.00 & 0.63 & 0.555 \\
\hline GUJ0087 & $151-155$ & 55 & 1.5 & 145 & 1 & 1.0 & 0.00 & 0.00 & 0.000 \\
\hline GUJ0091 & $172-188$ & 55 & 2.0 & $162-164$ & 2 & 1.3 & 0.30 & 0.26 & 0.222 \\
\hline GUJ0093 & $213-231$ & 60 & 2.0 & $218-224$ & 2 & 1.2 & 0.15 & 0.14 & 0.129 \\
\hline GUJ0094 & $237-249$ & 55 & 1.5 & 291 & 1 & 1.0 & 0.00 & 0.00 & 0.000 \\
\hline
\end{tabular}


Table II. Continued.

\begin{tabular}{lccccccccc}
\hline $\begin{array}{l}\text { Locus } \\
\text { name }\end{array}$ & $\begin{array}{c}\text { Size range } \\
\text { (bp) } \\
\text { in quail }\end{array}$ & $\begin{array}{c}T_{\mathrm{A}} \\
\left({ }^{\circ} \mathrm{C}\right)\end{array}$ & $\begin{array}{c}{\left[\mathrm{MgCl}_{2}\right]} \\
(\mathrm{mM})\end{array}$ & $\begin{array}{c}\text { Size range } \\
(\mathrm{bp}) \\
\text { in chicken }\end{array}$ & $N_{\mathrm{O}}$ & $N_{\mathrm{E}}$ & $H_{\mathrm{O}}$ & $H_{\mathrm{E}}$ & $P I C$ \\
\hline GUJ0097 & $131-157$ & 55 & 1.5 & $123-129$ & 3 & 2.1 & 0.30 & 0.53 & 0.468 \\
GUJ0098 & $197-205$ & 55 & 2.5 & $196-210$ & 4 & 2.2 & 0.75 & 0.54 & 0.483 \\
GUJ0099 & $246-284$ & 55 & 1.5 & $237-253$ & 2 & 1.7 & 0.10 & 0.42 & 0.332 \\
\hline
\end{tabular}

" Amplification products were obtained in 20 randomly sampled chicken using the annealing temperature optimized for quails.

$T_{\mathrm{A}}$, annealing temperature; $N_{\mathrm{O}}$, observed number of alleles; $N_{\mathrm{E}}$, effective number of alleles; $H_{\mathrm{O}}$, observed heterozygosity; $H_{\mathrm{E}}$, expected heterozygosity; PIC, polymorphism information content.

* Loci for which sequences were determined.

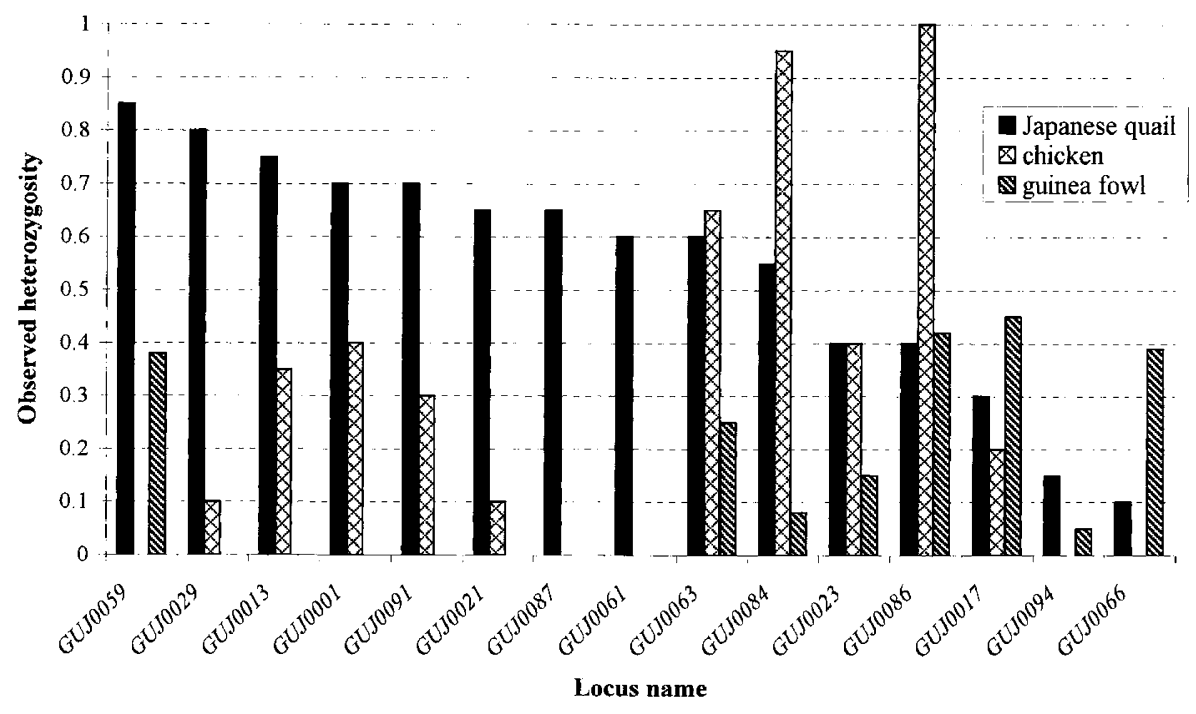

Figure 1. Observed heterozygosity in Japanese quail, chickens, and guinea fowl for the 15 quail markers found to cross-amplify DNA from the two other species. Observed heterozygosities of the 15 cross-reactive quail markers were estimated in random samples of 20 Japanese quail, 20 chickens, and 20 guinea fowls, each sample made up of 10 males and 10 females. The markers were ordered, from left to right, by decreasing heterozygosity in Japanese quail.

which were perfect repeats and 2 (GUC0001 and GUC0010) were imperfect repeats as found in their corresponding quail loci. For the remaining 2 loci, the repeat array was either perfect in the chicken, as opposed to imperfect (GUC0004), or vice versa (GUC0008) in the quail. The GUC0005 locus only had a poly A. Sequence alignment of the $5^{\prime}$ flanks of the corresponding quail 
Table III. Characteristics of 20 Japanese quail microsatellite loci amplified in guinea fowl ${ }^{\#}$.

\begin{tabular}{|c|c|c|c|c|c|c|c|c|c|}
\hline $\begin{array}{l}\text { Locus } \\
\text { name }\end{array}$ & $\begin{array}{c}\text { Size range } \\
\text { (bp) } \\
\text { in quail }\end{array}$ & $\begin{array}{c}T_{\mathrm{A}} \\
\left({ }^{\circ} \mathrm{C}\right)\end{array}$ & $\begin{array}{c}{\left[\mathrm{MgCl}_{2}\right]} \\
(\mathrm{mM})\end{array}$ & $\begin{array}{l}\text { Size range } \\
\text { (bp) } \\
\text { in guinea } \\
\text { fowl }\end{array}$ & $N_{\mathrm{O}}$ & $N_{\mathrm{E}}$ & $H_{\mathrm{O}}$ & $H_{\mathrm{E}}$ & $P I C$ \\
\hline GUJ0001 * & $231-239$ & 56 & 1.5 & 226 & 1 & 1.0 & 0.00 & 0.00 & 0.000 \\
\hline GUJ0013* & $127-139$ & 55 & 1.5 & 139 & 1 & 1.0 & 0.00 & 0.00 & 0.000 \\
\hline GUJ0017* & $153-165$ & 60 & 1.5 & $153-161$ & 3 & 2.7 & 0.45 & 0.63 & 0.550 \\
\hline GUJ0021 * & $143-157$ & 62 & 1.5 & 135 & 1 & 1.0 & 0.00 & 0.00 & 0.000 \\
\hline GUJ0023 & $219-237$ & 55 & 2.0 & $233-245$ & 3 & 1.2 & 0.15 & 0.14 & 0.140 \\
\hline GUJ0029* & $140-152$ & 55 & 1.5 & 130 & 1 & 1.0 & 0.00 & 0.00 & 0.000 \\
\hline GUJ0039 & $164-188$ & 60 & 2.0 & $159-163$ & 2 & 1.1 & 0.13 & 0.12 & 0.110 \\
\hline GUJ0040 & $176-192$ & 55 & 1.5 & 171 & 1 & 1.0 & 0.00 & 0.00 & 0.000 \\
\hline GUJ0059* & $207-219$ & 50 & 1.5 & $204-226$ & 4 & 1.5 & 0.38 & 0.33 & 0.311 \\
\hline GUJ0061 * & $157-171$ & 55 & 1.5 & 158 & 1 & 1.0 & 0.00 & 0.00 & 0.000 \\
\hline GUJ0063 & $242-250$ & 55 & 2.0 & $220-224$ & 2 & 2.0 & 0.25 & 0.50 & 0.374 \\
\hline GUJ0064 & $214-220$ & 55 & 2.0 & $220-224$ & 2 & 2.0 & 0.20 & 0.50 & 0.372 \\
\hline GUJ0066* & $167-175$ & 55 & 1.5 & 186-194 & 5 & 4.1 & 0.39 & 0.75 & 0.710 \\
\hline GUJ0073* & $144-160$ & 52 & 1.5 & $147-149$ & 2 & 1.0 & 0.04 & 0.04 & 0.040 \\
\hline GUJ0084* & $159-165$ & 55 & 1.5 & $168-170$ & 2 & 1.1 & 0.08 & 0.08 & 0.077 \\
\hline GUJ0086 & $197-207$ & 55 & 1.5 & $211-215$ & 2 & 2.0 & 0.42 & 0.50 & 0.373 \\
\hline GUJ0087 & $151-155$ & 55 & 1.5 & 137 & 1 & 1.0 & 0.00 & 0.00 & 0.000 \\
\hline GUJ0089 & $131-145$ & 55 & 2.5 & 123 & 1 & 1.0 & 0.00 & 0.00 & 0.000 \\
\hline GUJ0091 & $172-188$ & 55 & 2.5 & 165 & 1 & 1.0 & 0.00 & 0.00 & 0.000 \\
\hline GUJ0094 & $237-249$ & 55 & 1.5 & $313-317$ & 2 & 1.1 & 0.05 & 0.05 & 0.048 \\
\hline
\end{tabular}

\# Amplification products were obtained in 20 randomly sampled guinea fowls using the annealing temperature optimized for quails.

$T_{\mathrm{A}}$, annealing temperature; $N_{\mathrm{O}}$, observed number of alleles; $N_{\mathrm{E}}$, effective number of alleles; $H_{\mathrm{O}}$, observed heterozygosity; $H_{\mathrm{E}}$, expected heterozygosity; PIC, polymorphism information content.

* Loci for which sequences were determined.

and chicken loci revealed significant homologies ranging from $78.9 \%$ to $93.9 \%$. A BLAST search with sequences in GenBank showed no significant homology except for similarity with orthologous quail sequences that we had registered previously [19].

Table V shows the sequence results of 10 guinea fowl loci amplified by crossreactive quail markers. The sequence of 6 loci included $(\mathrm{CA} / \mathrm{GT})_{n}$ repeats. Two loci (GUG0006 and GUG0010) had perfect repeats and 2 (GUG0001 and $G U G 0008$ ) had imperfect repeats similar to their orthologous loci in the quail, while 2 loci ( $G U G 0002$ and $G U G 0003$ ) had imperfect repeats as opposed to the perfect repeats found in their corresponding quail loci. The remaining 4 guinea 
Table IV. Sequence results of 10 Japanese quail and chicken loci amplified by the same quail markers.

\begin{tabular}{|c|c|c|c|c|c|c|}
\hline \multicolumn{3}{|c|}{ Japanese quail } & \multicolumn{2}{|c|}{ Chicken } & \multirow[b]{2}{*}{ Repeat array } & \multirow[b]{2}{*}{$\begin{array}{l}\% \text { similarity } \\
\text { between Japanese } \\
\text { quail and chicken } \\
5^{\prime} \text { flank }\end{array}$} \\
\hline $\begin{array}{l}\text { Locus } \\
\text { name }\end{array}$ & $\begin{array}{l}\text { GenBank } \\
\text { accession } \\
\text { number }\end{array}$ & Repeat array & $\begin{array}{l}\text { Locus } \\
\text { name* }\end{array}$ & $\begin{array}{l}\text { GenBank } \\
\text { accession } \\
\text { number }\end{array}$ & & \\
\hline GUJ0001 & AB035652 & (CA)7TG(CA) 13 & GUC0001 & AB063261 & (CA)2CG(CA)3TG(CA)5GA(CA)11 & $84.1(176 \mathrm{nt})$ \\
\hline GUJ0013 & AB035823 & (CA)10 & GUC0002 & AB063262 & $(\mathrm{CA}) 5$ & 85.7 (91 nt) \\
\hline GUJ0017 & AB035827 & (CA) 14 & GUC0003 & AB063263 & $(\mathrm{CA}) 8$ & $93.9(98 \mathrm{nt})$ \\
\hline GUJ0023 & AB035833 & (CA)7TA(CA) 11 & GUC0004 & AB063264 & (CA) 17 & $78.9(152 \mathrm{nt})$ \\
\hline GUJ0029 & AB035839 & (CA) $11 \mathrm{CT}(\mathrm{CA}) 2$ & GUC0005 & AB063265 & (A) 14 & 92.7 (124 nt) \\
\hline GUJ0042 & AB035852 & (CA) 8 & GUC0006 & AB063266 & (CA)7 & $81.0(147 \mathrm{nt})$ \\
\hline GUJ0044 & AB035854 & (CA) 16 & GUC0007 & AB063267 & $(\mathrm{CA}) 3$ & 85.4 (123 nt) \\
\hline GUJ0049 & AB035859 & (CA) 11 & GUC0008 & AB063268 & $(\mathrm{CA}) 2 \mathrm{~A}(\mathrm{CA}) 5$ & 80.0 (200 nt) \\
\hline GUJ0059 & AB063127 & (CA) 10 & GUC0009 & AB063269 & (CA)11 & 82.7 (110 nt) \\
\hline GUJ0063 & AB063131 & (CA)7CT(CA)2CT(CA)7 & GUC0010 & AB063270 & (CA)6CC(CA)8 & 85.5 (138 nt) \\
\hline
\end{tabular}

* The locus code $G U C$ stands for Gifu University chicken and is in accordance with the standardized nomenclature rules adopted for poultry [5]. 
fowl loci had no repeat arrays. However, for all 10 loci, the sequences of the $5^{\prime}$ flanking regions were very similar to the corresponding quail sequences $(74.8 \%$ to $95.1 \%$ ). When searched against the database in GenBank, no matches were found for these sequences except our registered quail sequences.

\section{DISCUSSION}

The isolation of 50 new microsatellite markers in Japanese quail is a follow up on our earlier success in targeting simple sequence repeat (SSR) loci from an enriched genomic library [19] aimed at generating sufficient original quail markers for constructing a genetic map for this economically important poultry species. Previous attempts to localize quail SSR using chicken-specific primers have not been very successful. In one report [27], 22.9\% specific amplification was obtained from 48 chicken markers tested in quail but eventually only 6 markers were developed. In a related study [14], we could only amplify 31 (25.8\%) of 120 chicken microsatellite markers in Japanese quail, 22 of which were non-specific amplifications. This led us to the conclusion that chicken microsatellite primers are not efficient markers for Japanese quail, thereby underscoring the need to develop original markers for quail.

In our earlier report [19], 46.0\% (23/50) of the markers showed polymorphism in two unrelated quails. However, in this expanded study $98.0 \%(98 / 100)$ were polymorphic in 20 unrelated quails, thus clearly indicating that the larger sample size is more informative. Values of $75.8 \%$ (25/33) [6] and $93.2 \%$ (259/278) [7] polymorphisms have been reported for chicken-specific markers tested in the chicken. The very high level of polymorphism seen in the quail markers could, in part, be a reflection of the genetic constitution of the test population, which was derived from a colony of wild quail origin and is thus considered to be genetically diverse as a result of its shorter history of domestication [18]. The average number of alleles observed in the Japanese quail was 3.7, ranging from 1 to 6 . This is similar to a mean of 4 and a range of 2 to 9 [7] or a mean of 5.6 and a range of 2 to 10 [41] reported for the chicken. Based on the PIC values, nearly $60.0 \%$ of the polymorphic markers were highly informative and only a few (12.2\%) were slightly informative. Therefore, we conclude that these markers have a high utility for mapping the quail genome.

As a step towards constructing a comparative genetic map in the Phasianidae family, which includes a number of agriculturally important species of poultry, cross-species amplification was carried out to determine the usefulness of Japanese quail markers in chicken and guinea fowl. The level of amplification observed in the chicken in the present study $(42.0 \%)$ is consistent with the results of other studies of cross-species amplification involving chicken markers applied to turkeys $(91.7 \%$ [21], 51.1\% [22], 55.6\% [13], 55.3\% [32], and $53.8 \%$ [33] specific amplifications), or chicken markers tested in the Japanese 
Table V. Sequence results of 10 Japanese quail and guinea fowl loci amplified by the same quail markers.

\begin{tabular}{|c|c|c|c|c|c|c|}
\hline \multicolumn{3}{|c|}{ Japanese quail } & \multicolumn{2}{|c|}{ Guinea fowl } & \multirow[b]{2}{*}{ Repeat array } & \multirow[b]{2}{*}{$\begin{array}{l}\% \text { similarity } \\
\text { between Japanese } \\
\text { quail and guinea } \\
\text { fowl } 5^{\prime} \text { flank }\end{array}$} \\
\hline $\begin{array}{l}\text { Locus } \\
\text { name }\end{array}$ & $\begin{array}{l}\text { GenBank } \\
\text { accession } \\
\text { number }\end{array}$ & Repeat array & $\begin{array}{l}\text { Locus } \\
\text { name* }\end{array}$ & $\begin{array}{l}\text { GenBank } \\
\text { accession } \\
\text { number }\end{array}$ & & \\
\hline GUJ0001 & AB035652 & (CA)7TG(CA)13 & GUG0001 & AB063271 & (CA)2CG(CA)12 & $83.1(148 \mathrm{nt})$ \\
\hline GUJ0013 & AB035823 & (CA) 10 & GUG0002 & AB063272 & (CA)7CC(A)19 & $81.9(83 \mathrm{nt})$ \\
\hline GUJ0017 & AB035827 & (CA) 14 & GUG0003 & AB063273 & $(\mathrm{CA}) 2(\mathrm{~A}) 20$ & $87.3(134 \mathrm{nt})$ \\
\hline GUJ0021 & AB035831 & (CA)11 & GUG0004 & AB063274 & $\mathrm{X}$ & 83.7 (135 nt) \\
\hline GUJ0029 & AB035839 & (CA)11CT(CA)2 & GUG0005 & AB063275 & $\mathrm{X}$ & 85.5 (124 nt) \\
\hline GUJ0059 & AB063127 & $(\mathrm{CA}) 10$ & GUG0006 & AB063276 & (CA) 11 & 84.7 (196 nt) \\
\hline GUJ0061 & AB063129 & (CA) 15 & GUG0007 & AB063277 & $\mathrm{X}$ & $87.8(90 \mathrm{nt})$ \\
\hline GUJ0066 & AB063134 & (CA) $12 \mathrm{TA}(\mathrm{CA}) 2$ & GUG0008 & AB063278 & (CA)27CG(CA)2CG(CA)5 & 74.8 (135 nt) \\
\hline GUJ0073 & AB063141 & $(\mathrm{CA}) 13$ & GUG0009 & AB063279 & $\mathrm{X}$ & 79.6 (142 nt) \\
\hline GUJ0084 & AB063152 & (CA) 10 & GUG0010 & AB063280 & (CA) 12 & 95.1 (143 nt) \\
\hline
\end{tabular}

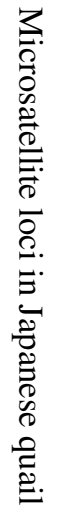

* The locus code GUG stands for Gifu University guinea fowl and is in accordance with the standardized nomenclature rules adopted for poultry [5].

$\mathrm{X}$, No repeats detected. 
quail (22.9\% [27] and 25.8\% [14] specific PCR products). Although we adjusted the $\mathrm{MgCl}_{2}$ concentration, we did not attempt to optimize the amplification condition for any locus. Hence, it is likely that such an effort would yield more positive amplifications. In our earlier study using chicken primers on quail, no adjustment was made in the $\mathrm{MgCl}_{2}$ concentration, and this could partly account for the lower amplification success of $25.8 \%$ [14]. The average observed number of alleles for quail markers tested in the chicken was 1.9. This value is lower than the 3.7 number of alleles observed for quail in this study, but is, however, close to the value of 1.4 reported for chicken markers tested in turkeys [33]. The lower value of the number of alleles observed in chickens as compared to quail could, in part, be due to the characteristics of the test populations, since wild-derived quail were used on the one hand and White Leghorn chickens on the other. However, studies on cross-reactive markers have shown that microsatellite repeats tend to be generally longer, and thus more polymorphic, in the species of origin than in the comparison species, thus suggesting an ascertainment bias $[10,33]$. This could have also contributed to the differences observed. From the PIC data, the polymorphic cross-reactive markers were reasonably informative and would be useful for comparative mapping in chickens and Japanese quail.

In guinea fowl, 20 of the quail markers amplified loci, with the observed number of alleles per locus averaging 1.9, and 11 of them were polymorphic. Although the mean observed number of alleles per locus was similar to that in chickens, the mean observed heterozygosity and PIC were lower in guinea fowl. This is particularly evident in Figure 1 for the 15 markers that crossamplified Japanese quail, chicken and guinea fowl DNA. Apart from the possible ascertainment bias mentioned earlier, one reason for this might be due to the low heterogeneity suspected in the guinea fowl population that was sampled, since it is probable that only a very small number of founders were introduced into Japan as is evidenced by the few guinea fowl farms that exist. In spite of this, a considerable number of the cross-reactive markers in guinea fowl are reasonably informative and would be useful for comparative mapping.

Out of the 15 markers cross-reacting in Japanese quail, chickens and guinea fowl, five markers (GUJ0017, GUJ0023, GUJ0063, GUJ0084, and GUJ0086) were informative in our test populations and would thus serve as the backbone of a comparative map in these Phasianidae species. Although the remaining 10 markers were not polymorphic in all three species, it is likely that they would be polymorphic when tested in a larger population, or they could be useful in the future as markers for radiation hybrid mapping [20].

By sequencing PCR products of a random sample of the cross-reactive markers, we observed that all the markers shared sequence identity with the quail ( $>78.9 \%$ in chicken and $>74.8 \%$ in guinea fowl). Nine out of 10 sequences in chickens included $(\mathrm{CA} / \mathrm{GT})_{n}$ microsatellites compared to 6 out 
of the 10 guinea fowl sequences. Similar observations have been made in other studies on cross-species amplification involving chicken markers in quail in which 2 out of 10 loci [27] and three out of 9 loci [14] sequenced had no microsatellites. In this study, three of the guinea fowl sequences lacking microsatellites were not polymorphic. The greater number of quail markers that amplified chicken DNA as opposed to guinea fowl DNA, and the higher similarity of the quail-chicken flanking sequences compared to the quail-guinea fowl sequences, coupled with a better conservation of microsatellite loci in orthologous quail-chicken sequences than quail-guinea fowl sequences, are useful observations pointing to a closer relation between quail and chickens and could thus contribute to the discussion on the phylogenetic relationship of the three species. However, our data was limited and therefore inconclusive in this regard. Studies on phyletic relationships based on homologies of chromosome banding patterns have placed Gallus, Coturnix and Numida in the same subfamily, with Coturnix and Gallus being more closely related than Numida and Gallus [39]. It has been recently confirmed that chromosome homology between Japanese quail and chickens is highly conserved, with very few chromosome rearrangements after divergence of the two species (Matsuda Y., personal communication). Sequencing and microsatellite genotyping data based on cross-reactive markers in quail, chickens, and guinea fowl could, therefore complement our understanding of the phylogenetic relationships between these species.

From this study, we report $9(\mathrm{CA} / \mathrm{GT})_{n}$ microsatellite-containing quail markers as new markers for chickens. Similarly, six quail markers are being reported as the first novel microsatellite markers registered for guinea fowl. The guinea fowl has been reputed to be a species with great potential, able to adapt easily to all kinds of climate in spite of its African origin [25]. In view of this, DNA markers for this species would help promote their genetic improvement. Based on our results, we recommend the isolation of original microsatellite markers for mapping in guinea fowl rather than attempting to adapt markers isolated from other species for studies in guinea fowl.

In conclusion, we have described informative Japanese quail microsatellite markers that would form a useful resource base of DNA markers as part of our initiative to develop a genetic map for Japanese quail. Since crossspecies amplification indicated that several of the cross-reactive markers are informative in chickens (57.1\%) and guinea fowl (55.0\%), these markers may be useful for comparative genome analysis in Phasianidae. Furthermore, the crossreactive markers could be used as a tool in future phylogenetic studies aimed at improving our understanding of the relatedness of Japanese quail to chickens and guinea fowl. The trend in comparative mapping in poultry is taking several directions including the analysis of cDNA clones [38] and radiation hybrid mapping [20], and our results would contribute to this collective effort. 


\section{ACKNOWLEDGEMENTS}

We gratefully acknowledge the dedicated technical assistance of Ms. Y. Ueda, whose efforts greatly aided this work. The blood samples from guinea fowls were kindly supplied by Mr. J. Ninomiya, President, JAFRA TRADING CO., LTD., Japan, to whom we are most thankful. This research was financially supported by the Japan Livestock Technology Association.

\section{REFERENCES}

[1] Botstein D., White R.L., Skolnick M., Davis R.W., Construction of a genetic linkage map in man using restriction fragment length polymorphisms, Am. J. Hum. Genet. 32 (1980) 314-331.

[2] Bumstead N., Palyga J., A preliminary linkage map of the chicken genome, Genomics 13 (1992) 690-697.

[3] Crawford A.M., Dodds K.G., Ede A.J., Pierson C.A., Montgomery G.W., Garmonsway H.G., Beattie A.E., Davies K., Maddox J.F., Kappes S.W., Stone R.T., Nguyen T.C., Penty J.M., Lord E.A., Broom J.E., Buitkamp J., Schwaiger W., Epplen J.T., Matthew P., Matthews M.E., Hulme D.J., Beh K.J., McGraw R.A., Beattie C.W., An autosomal genetic linkage map of the sheep genome, Genetics 140 (1995) 703-724.

[4] Crittenden L.B., Provencher L., Santangelo L., Levin I., Abplanalp H., Briles R.W., Briles W.E., Dodgson J.B., Characterization of a Red Jungle Fowl by White Leghorn backcross reference population for molecular mapping of the chicken genome, Poultry Sci. 72 (1993) 334-348.

[5] Crittenden L.B., Bitgood J.J., Burt D.W., Ponce de Leon F.A., Tixier-Boichard M., Nomenclature for naming loci, alleles, linkage groups and chromosomes to be used in poultry genome publications and databases, Genet. Sel. Evol. 28 (1996) 289-297.

[6] Crooijmans R.P.M.A., van der Poel J.J., Groenen M.A.M., Functional genes mapped on the chicken genome, Anim. Genet. 26 (1995) 73-78.

[7] Crooijmans R.P.M.A., Dijkhof R.J.M., van der Poel J.J., Groenen M.A.M., New microsatellite markers in chicken optimized for automated fluorescent genotyping, Anim. Genet. 28 (1997) 427-437.

[8] Dawson R.J., Gibbs H.L., Hobson K.A., Yezerinac S.M., Isolation of microsatellite DNA markers from a passerine bird, Dendroica petechia (the yellow warbler), and their use in population studies, Heredity 79 (1997) 506-514.

[9] de Gortari M.J., Freking B.A., Kappes S.M., Leymaster K.A., Crawford A.M., Stone R.T., Beattie C.W., Extensive genomic conservation of cattle microsatellite heterozygosity in sheep, Anim. Genet. 28 (1997) 274-290.

[10] Ellegren H., Moore S., Robinson N., Byrne K., Ward W., Sheldon B.C., Microsatellite evolution - a reciprocal study of repeat lengths at homologous loci in cattle and sheep, Mol. Biol. Evol. 14 (1997) 854-860. 
[11] Groenen M.A.M., Crooijmans R.P.M.A., Veenendaal A., Cheng H.H., Siwek M., van der Poel J.J., A comprehensive microsatellite linkage map of the chicken genome, Genomics 49 (1998) 265-274.

[12] Groenen M.A.M., Cheng H.H., Bumstead N., Benkel B.F., Briles W.E., Burke T., Burt D.W., Crittenden L.B., Dodgson J., Hillel J., Lamont S., Ponce de Leon A., Soller M., Takahashi H., Vignal A., A consensus linkage map of the chicken genome, Genome Res. 10 (2000) 137-147.

[13] Hanotte O., Pugh A., Maücher C., Dawson D., Burke T., Nine novel chicken microsatellite loci and their utility in other Galliformes, Anim. Genet. 28 (1997) 311-313.

[14] Inoue-Murayama M., Kayang B.B., Kimura K., Ide H., Nomura A., Takahashi H., Nagamine Y., Takeda T., Hanada H., Tatsuda K., Tsudzuki M., Matsuda Y., Mizutani M., Murayama Y., Ito S., Chicken microsatellite primers are not efficient markers for Japanese quail, Anim. Genet. 32 (2001) 7-11.

[15] Ito S., Kimura M., Isogai I., A sex difference in recombination values between extended brown and phosphoglucose isomerase loci in Japanese quail, Jpn. J. Zootech. Sci. 59 (1988) 801-805.

[16] Ito S., Kimura M., Isogai I., Linkage between panda plumage and albumin loci in Japanese quail, Jpn. J. Zootech. Sci. 59 (1988) 822-824.

[17] Kappes S.M., Keele J.W., Stone R.T., McGraw R.A., Sonstegard T.S., Smith T.P.L., Lopez-Corrales N.L., Beattie C.W., A second-generation linkage map of the bovine genome, Genome Res. 7 (1997) 235-249.

[18] Kawahara T., Comparative study of quantitative traits between wild and domestic Japanese quail (Coturnix coturnix japonica), Exp. Anim. 22 (Suppl.) (1973) 139150.

[19] Kayang B.B., Inoue-Murayama M., Nomura A., Kimura K., Takahashi H., Mizutani M., Ito S., Fifty microsatellite markers for Japanese quail, J. Hered. 91 (2000) 502-505.

[20] Kwok C., Korn R.M., Davis M.E., Burt D.W., Critcher R., McCarthy L., Paw B.H., Zon L.I., Goodfellow P.N., Schmitt K., Characterization of whole genome radiation hybrid mapping resources for non-mammalian vertebrates, Nucleic Acids Res. 26 (1998) 3562-3566.

[21] Levin I., Cheng H.H., Baxter-Jones C., Hillel J., Turkey microsatellite DNA loci amplified by chicken-specific primers, Anim. Genet. 26 (1995) 107-110.

[22] Liu Z., Crooijmans R.P.M.A., van der Poel J.J., Groenen M.A.M., Use of chicken microsatellite markers in turkey: a pessimistic view, Anim. Genet. 27 (1996) 191-193.

[23] Minvielle F., Genetics and breeding of Japanese quail for production around the world, in: Proceedings of the 6th Asian Pacific Poultry Congress, Nagoya, Japan, 4-7 June 1998, Japan Poultry ScienceAssociation, Tsukuba, pp. 122-127.

[24] Minvielle F., Ito S., Inoue-Murayama M., Mizutani M., Wakasugi N., Genetic analyses of plumage color mutations on the $\mathrm{Z}$ chromosome of Japanese quail, J. Hered. 91 (2000) 499-501. 
[25] Mongin P., Plouzeau M., Guinea fowl, in: Mason I.L. (Ed.), Evolution of Domesticated Animals, Longman, London, 1984, pp. 322-325.

[26] Padgett C.A., Ivey W.D., Coturnix quail as a laboratory research animal, Science 129 (1959) 267-268.

[27] Pang S.W.Y., Ritland C., Carlson J.E., Cheng K.M., Japanese quail microsatellite loci amplified with chicken-specific primers, Anim. Genet. 30 (1999) 195-199.

[28] Pépin L., Amigues Y., Lépingle A., Berthier J.-L., Bensaid A., Vaiman D., Sequence conservation of microsatellites between Bos taurus (cattle), Capra hircus (goat) and related species. Examples of use in parentage testing and phylogeny analysis, Heredity 74 (1995) 53-61.

[29] Petren K., Microsatellite primers from Geospiza fortis and cross-species amplification in Darwin's finches, Mol. Ecol. 7 (1998) 1782-1784.

[30] Primmer C.R., Møller A.P., Ellegren H., A wide-range survey of cross-species microsatellite amplification in birds, Mol. Ecol. 5 (1996) 365-378.

[31] Primmer C.R., Raudsepp T., Chowdhary B.P., Møller A.P., Ellegren H., Low frequency of microsatellites in the avian genome, Genome Res. 7 (1997) 471482.

[32] Reed K.M., Mendoza K.M., Beattie C.W., Utility of chicken-specific microsatellite primers for mapping the turkey genome, Anim Biotechnol. 10 (1999) 137141.

[33] Reed K.M., Mendoza K.M., Beattie C.W., Comparative analysis of microsatellite loci in chicken and turkey, Genome 43 (2000) 796-802.

[34] Richardson D.S., Jury F.L., Dawson D.A., Salgueiro P., Komdeur J., Burke T., Fifty Seychelles warbler (Acrocephalus sechellensis) microsatellite loci polymorphic in Sylviidae species and their cross-species amplification in other passerine birds, Mol. Ecol. 9 (2000) 2226-2231.

[35] Rohrer G.A., Alexander L.J., Hu Z., Smith T.P.L., Keele J.W., Beattie C.W., A comprehensive map of the porcine genome, Genome Res. 6 (1996) 371-391.

[36] Shibata T., Abe T., Linkage between the loci for serum albumin and vitamin D binding protein (GC) in the Japanese quail, Anim. Genet. 27 (1996) 195-197.

[37] Slate J., Coltman D.W., Goodman S.J., MacLean I., Pemberton J.M., Williams J.L., Bovine microsatellite loci are highly conserved in red deer (Cervus elaphus), sika deer (Cervus nippon) and Soay sheep (Ovis aries), Anim. Genet. 29 (1998) 307-315.

[38] Smith E., Shi L., Drummond P., Rodriguez L., Hamilton R., Powell E., Nahashon S., Ramlal S., Smith G., Foster J., Development and characterization of expressed sequence tags for the turkey (Meleagris gallopavo) genome and comparative sequence analysis with other birds, Anim. Genet. 31 (2000) 62-67.

[39] Stock A.D., Bunch T.D., The evolutionary implications of chromosome banding pattern homologies in the bird order Galliformes, Cytogenet. Cell Genet. 34 (1982) 136-148. 
[40] Takahashi H., Nirasawa K., Furukawa T., An efficient method to clone chicken microsatellite repeat sequences, Jpn. Poult. Sci. 33 (1996) 292-299.

[41] Takahashi H., Nirasawa K., Nagamine Y., Tsudzuki M., Yamamoto Y., Genetic relationships among Japanese native breeds of chicken based on microsatellite DNA polymorphisms, J. Hered. 89 (1998) 543-546.

[42] Vaiman D., Schibler L., Bourgeois F., Oustry A., Amigues Y., Cribiu E.P., A genetic linkage map of the male goat genome, Genetics 144 (1996) 279-305.

[43] Wakasugi N., Kondo K., Breeding methods for maintenance of mutant genes and establishment of strains in the Japanese quail, Exp. Anim. 22 (Suppl.) (1973) 151-159.

[44] Weber J.L., Informativeness of human (dC-dA)n.(dG-dT)n polymorphisms, Genomics 7 (1990) 524-530.

[45] Wilson W.O., Abbott U.K., Abplanalp H., Evaluation of Coturnix (Japanese quail) as pilot animal for poultry, Poultry Sci. 40 (1961) 651-657.

To access this journal online: www.edpsciences.org 